\title{
Multi-hybrid Active Magnetic Bearing Design for Milling Spindle Applications
}

\author{
Rong-Mao Lee, ${ }^{1 *}$ Zhi-Bin Wu, ${ }^{2}$ Cheng-Chi Wang, ${ }^{3}$ and Tsung-Chia Chen ${ }^{2}$ \\ ${ }^{1}$ Computer and Intelligent Robot Program for Bachelor Degree, National Pingtung University, \\ No. 4-18, Minsheng Rd., Pingtung City, Pingtung County 90003, Taiwan \\ ${ }^{2}$ Department of Mechanical Engineering, National Chin-Yi University of Technology, \\ No. 57, Sec. 2, Zhongshan Rd., Taiping Dist., Taichung City 41170, Taiwan \\ ${ }^{3} \mathrm{Ph}$.D. Program, Graduate Institute of Precision Manufacturing, National Chin-Yi University of Technology, \\ No. 57, Sec. 2, Zhongshan Rd., Taiping Dist., Taichung City 41170, Taiwan
}

(Received February 15, 2019; accepted November 21, 2019)

Keywords: active magnetic bearing, magnetic loop analysis, coupled magnetic flux, milling spindle

Active magnetic bearings (AMBs) have been gradually developed and used in industrial applications. For a milling spindle system with full magnetic support, at least one axial AMB and two radial AMBs should be included. As a result, the milling spindle dimensions will probably be too large for the application of machine tools. A multi-hybrid AMB design, in which one axial $\mathrm{AMB}$, one radial $\mathrm{AMB}$, and a permanent magnet (PM) are combined, is reported in this work. The PM is embedded for axial suspension for the rotor weight so that the force requirement and the dimensions of the axial AMB can be reduced. On the other hand, magnetic channels were specially designed to couple the three magnetic loops generated by the AMBs and PM instead of isolating these magnetic fluxes individually. The proposed design is also suitable for applications of robot arm joints, such as waist and shoulder joints, to improve the bearing lifetime under heavy loads and to actively regulate the joint position deviation. The proposed multi-hybrid AMB design was practically manufactured after intensive analyses and verified by practical tests.

\section{Introduction}

High-speed machining (HSM) is a long-term goal for the development of machine tools. Manufacturing precision can be improved with the aid of computer-aided manufacturing (CAM) software. However, increasing the machining speed still depends on improving the machine tool hardware, i.e., the driving motor, controller, and bearings. ${ }^{(1)}$ This work is focused on a novel bearing design. For a HSM spindle, the bearing is usually the bottleneck preventing a higher spindle speed, while maintaining the manufacturing precision and spindle lifetime. A contact bearing, such as a ball bearing, cannot easily overcome these bottleneck issues while increasing the spindle speed. As an alternative solution, active magnetic bearings (AMBs)

*Corresponding author: e-mail: maxmou@mail.nptu.edu.tw

https://doi.org/10.18494/SAM.2020.2600 
have been increasingly employed in transmission components or rotary devices, such as highspeed motors and pumps. AMBs are suitable for high-speed spindles owing to the non-contact operation mode and the capability of regulating the rotor position deviation. ${ }^{(2)}$

Noh et al. proposed a bearingless motor design. ${ }^{(3)}$ The rotor was radially biased with the flux generated by a permanent magnet (PM), on which the stator can superimpose two-pole flux to generate suspension forces. The two tilting degrees of freedom (DOF) and the axial translation were passively stable owing to the reluctance forces generated by the bias flux. Since the operation of the AMB and that of the motor were both triggered by the magnetic flux, their dynamics may be coupled when AMBs are employed to replace contact bearings inside the motor. The multiobjective genetic algorithm (GA) was synthesized for the AMB design method by Smirnov et al. to reduce the magnetic influence on the high-speed electrical machine performance. $^{(4)}$ A hybrid magnetic bearing (HMB) design to decrease the AMB power consumption in which PMs were used to increase the radial stability of the rotor, was reported. ${ }^{(5)}$ Since the AMB operation is highly nonlinear and time varying with external interference, a nonlinear adaptive sliding-mode controller was designed to control the position of the rotor. Shuyue et al. introduced a 5-DOF AMB and applied it to a helium centrifugal cold compressor. ${ }^{(6)}$ A control strategy combining proportional-integral-derivative (PID) arithmetic with other phase compensators was proposed to stabilize an AMB system and also guarantee good performance of the compressor. A novel design for an axial flow blood pump was proposed by Zad et al. in 2018. ${ }^{(7)}$ The main structure was designed according to the concept of a bearingless PM motor, and one radial AMB was included to generate the motor torque. For low-temperature applications, a superconducting magnetic bearing (SMB) has been designed and applied to a cryogenic liquid pump to reduce the disadvantages of conventional bearings, such as the difficulty in lubrication and a short working life. ${ }^{(8)}$

For machine tool applications, a new repulsive magnetic bearing system was designed by Mukhopadhyay; ${ }^{(9)}$ in this system, the deviation of the radial rotor position was regulated passively by the repulsive force of PMs, namely, the axial rotor position deviation could be controlled. A compact 5-axis AMB system with solid cores having a total of four PMs, four U-shaped cores, and 16 coils was proposed in 2006. ${ }^{(10)}$ The homopolar configuration was levitated by a Lorentz-type axial force as well as a Maxwell-type radial force. A miniature milling spindle with AMBs, in which reluctance actuators and PMs were used to accomplish a 5-DOF rotor, was reported in 2010. ${ }^{(11)}$ A model of rotor dynamics was developed for the controller design and a maximum speed of $150000 \mathrm{rpm}$ was achieved. To increase the magnetic force per unit volume of the AMB, an embedded cylindrical-array magnetic actuator (ECAMA) was designed. ${ }^{(12)}$ The coils were wound along the axial direction so that the space for each coil could be greatly increased. In other words, a stronger magnetic force could be induced without significantly increasing the AMB size. A motorized spindle supported by AMBs has been developed for ultrahigh-speed machining. ${ }^{(13)}$ To consider the thermal deformation due to iron loss at a high rotation speed, the influences of homopolar and heteropolar AMBs on spindle dynamics were studied. A conical AMB with a claw structure was proposed in 2014. ${ }^{(14)}$ The stator core and coils were located along the axial direction to obtain a small radial extension, and the axial and radial rotor position regulation functions were merged in a single 
magnetic structure for use when radial space is limited. A similar design, where a 5-DOF rotor system with conical AMBs was presented, was reported in 2017. ${ }^{(15)}$ By taking the magnetic path reluctance into consideration in the AMB design, the axial space can be reduced by approximately $37 \%$. A high-speed solid cylindrical PM machine design was proposed by Xu et $a l ;{ }^{(16)}$ in this design, AMBs were integrated to support the rotor. Since the PM machine and the AMB are both driven by magnetic loops, mutually related factors such as the eddy-current loss, the power density, and the coupling of magnetic fluxes were all investigated to determine the machine structure. A magnetic channel was specially designed to couple the axial and radial magnetic loops. As a result, the axial magnetic force was increased owing to their partial overlap in the magnetic channel. In other words, the coupled magnetic loops can be beneficial for system performance as long as the magnetic channels are properly designed, for example, the coupled loops are divided into major and minor loops.

The purpose of this work is to design and manufacture a multi-hybrid AMB, in which the regulation functions for both the radial and axial directions are combined. For the design of a 5-DOF maglev levitation milling spindle, at least two radial AMBs and one axial AMB must be included. The overall size of the maglev levitation milling spindle can be reduced by integrating one axial AMB and one radial AMB. The combination of AMBs in this work is not merely the design of individual magnetic structures but also the coupled magnetic channel. In addition, a PM was equipped to further reduce the AMB dimensions and power consumption.

\section{Design and Analysis of Multi-hybrid AMB}

Since the proposed AMB is for milling spindle and robot arm applications, a cylindricalarray structure ${ }^{(12)}$ was employed owing to its high magnetic force per unit volume. The standard cylindrical-array AMB for radial direction regulation is shown in Fig. 1. The design steps are (i) the design and analysis of a radial AMB, (ii) the design and analysis of an axial AMB, (iii) the design and analysis of magnetic channels for coupled magnetic fluxes.
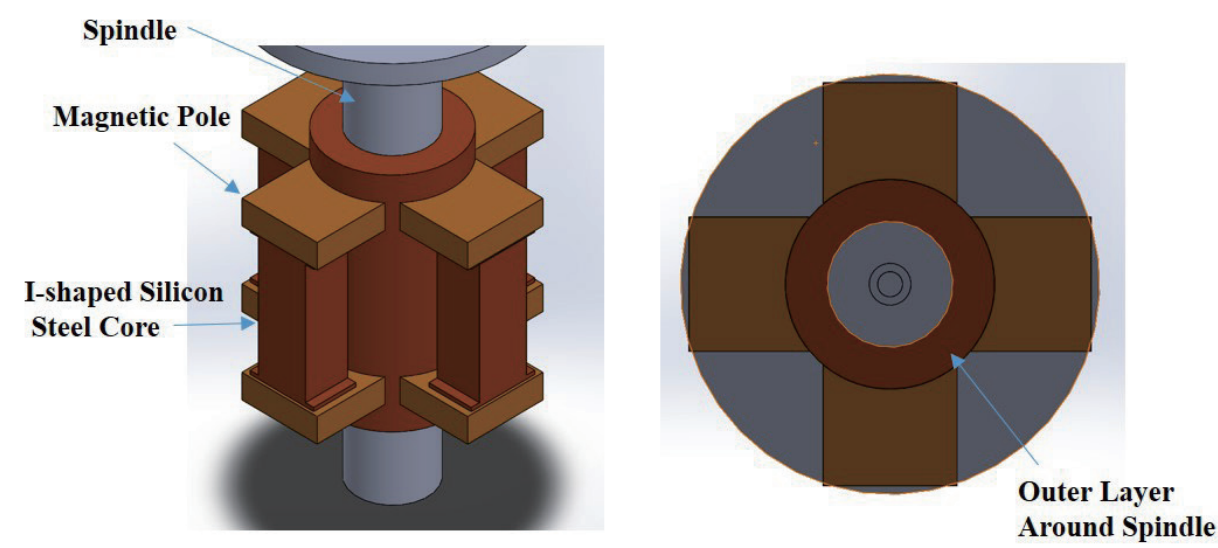

Fig. 1. (Color online) Structure of cylindrical-array AMB. 


\subsection{Design and analysis of radial AMB}

The radial AMB structure is shown in Fig. 1. The material properties of the components subjected to magnetic analyses are listed in Table 1. Partial AMB analysis results and some illustrations of results of magnetic flux density analysis are provided in Table 2 and Fig. 2, respectively. The goal of these magnetic analyses is to determine the relative dimensions of each component to avoid magnetic saturation.

\subsection{Design and analysis of axial AMB}

The proposed structure of the axial AMB is shown in Fig. 3. A PM was embedded to provide a lifting force to support the rotor weight so that the power consumption of the axial

Table 1

Material properties of components subjected to radial AMB analyses.

\begin{tabular}{lc}
\hline Component & Material property \\
\hline Silicon steel layer on spindle & Silicon steel 35H440 \\
Magnetic pole & Silicon steel 35H440 \\
I-shaped silicon steel core & Silicon steel 35H440 \\
Spindle & Steel 1010 \\
\hline
\end{tabular}

Table 2

Partial radial AMB analysis results.

\begin{tabular}{lccccc}
\hline No. & 1 & 2 & 3 & 4 & 5 \\
\hline Pole area against spindle $\left(\mathrm{mm}^{2}\right)$ & 66.1 & 66.1 & 66.1 & 100 & 130 \\
Pole thickness along axial direction $(\mathrm{mm})$ & 20 & 20 & 20 & 10 & 10 \\
Cross-sectional area of I-shaped core $\left(\mathrm{mm}^{2}\right)$ & $4.5 \times 4.5$ & $8 \times 8$ & $8 \times 8$ & $8 \times 8$ & $8 \times 8$ \\
Spindle diameter $(\mathrm{mm}) * \mathrm{~A}$ & 8 & 8 & 8 & 8 & 8 \\
Outer diameter of AMB $(\mathrm{mm})$ & 25 & 25 & 25 & 25 & 25 \\
Induced magnetic force $(\mathrm{N}) * \mathrm{~B}$ & $4.7(1 \mathrm{~A})$ & $12.6(1 \mathrm{~A})$ & $46(2 \mathrm{~A})$ & $60(1 \mathrm{~A})$ & $80(1 \mathrm{~A})$ \\
\hline
\end{tabular}

*A: includes the thickness of the silicon steel layer. *B: for single I-shaped silicon steel core with wound coil of 350 turns.

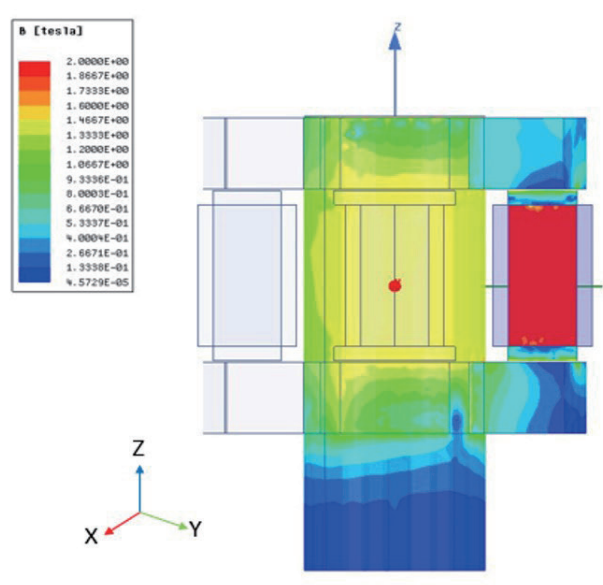

(a)

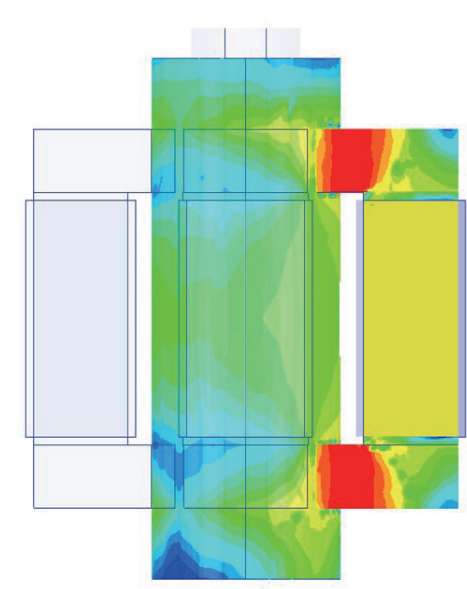

(b)

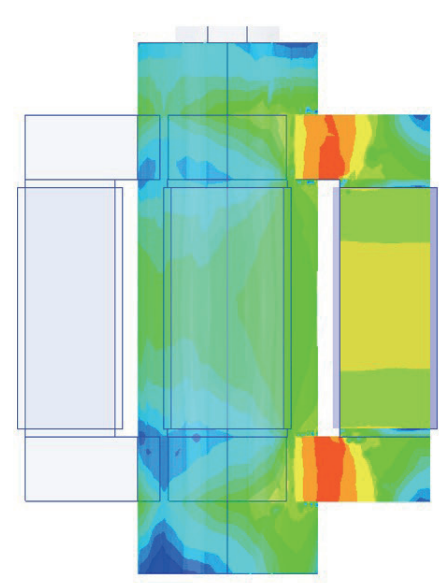

(c)

Fig. 2. (Color online) Illustrations of results of magnetic flux density analysis: (a) case No. 1, (b) case No. 2, and (c) case No. 5. 


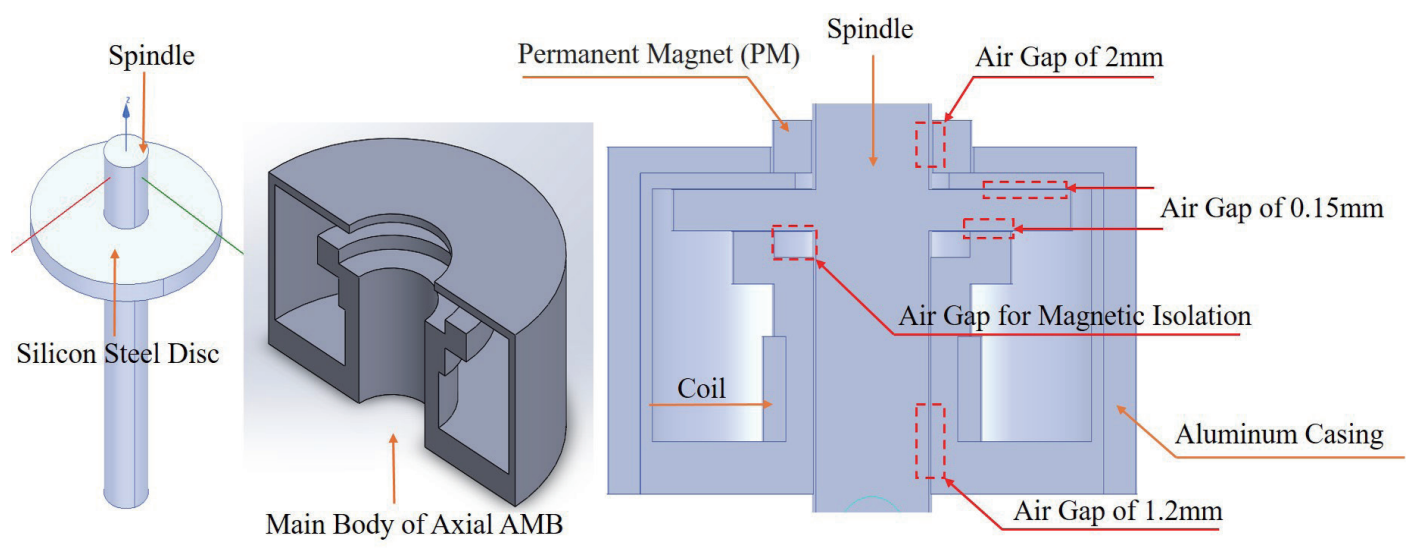

Fig. 3. (Color online) Proposed structure of axial AMB.

Table 3

Material properties for axial AMB analyses.

\begin{tabular}{lc}
\hline Component & Material \\
\hline Main body of axial AMB & Silicon steel 35H440 \\
Coil & Copper \\
Aluminum casing & Aluminum \\
Spindle & Steel 1010 \\
PM & NdFeB magnet \\
\hline
\end{tabular}

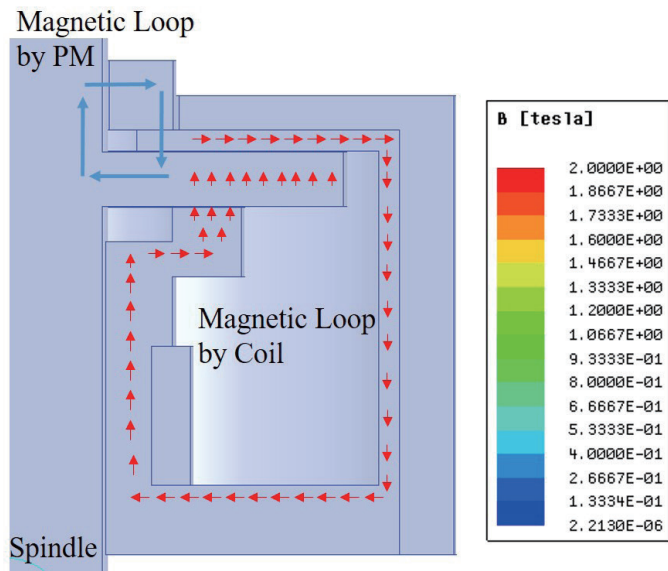

(a)

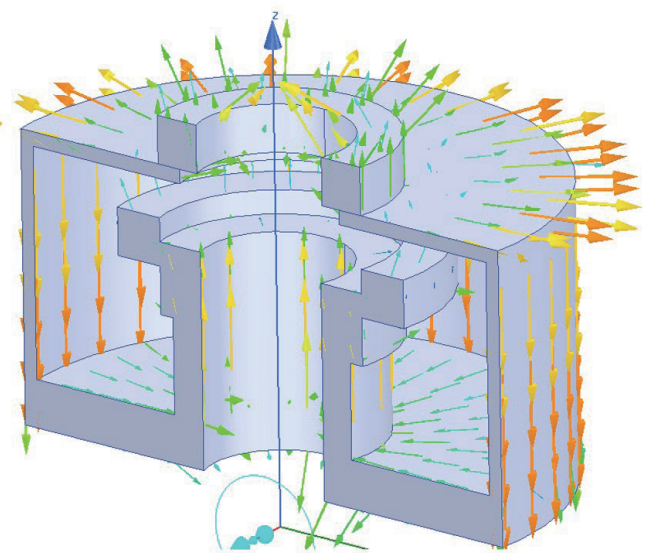

(b)

Fig. 4. (Color online) Magnetic analysis of axial AMB: (a) supposed magnetic loops and (b) analysis result.

coil can be reduced. The magnitude of the air gaps was mainly determined by considering the magnetic flux density. The material properties of the components subjected to magnetic analyses are listed in Table 3. The result of a magnetic loop analysis is illustrated in Fig. 4. In this case, the rotor weight is about $1.5 \mathrm{~kg}$, the lifting force by the PM (outer/inner diameters: $30 \mathrm{~mm} / 9 \mathrm{~mm}$ ) is $50 \mathrm{~N}$, and the magnetic force applied by the axial AMB at 350 ampere-turns is $108 \mathrm{~N}$. Since the magnetic loops generated by the PM and coil are coupled in the silicon 
steel disc on the spindle, the resulting axial lifting force can be regulated by the magnitude and direction of the axial coil current. The air gap for magnetic isolation is to ensure a uniform magnetic flux density inside the silicon steel disc and to prevent magnetic saturation in the spindle.

\subsection{Design and analysis of coupled magnetic channel for axial and radial AMBs}

As mentioned in Sect. 1, for coupled magnetic loops, the magnetic loops should be properly divided into major and minor loops. Two situations are discussed for our design, as shown in Fig. 5. The directions of magnetic fluxes depend on the coil current direction. There are one coil and four coils in the axial and radial AMBs, respectively. In Fig. 5(a), the minor loop receives part of the magnetic flux from the major loop. However, coil currents are supplied according to control demand and at most two radial coils (along different axes) will be activated at the same time. That means that the magnetic flux from the axial AMB will become a severe disturbance for rotor position control in the radial direction. Under the other condition shown in Fig. 5(b), the magnetic flux from the radial AMB can also be regarded as a disturbance for axial AMB operation. However, the axial control of rotor dynamics (only one axis) is much simpler than radial rotor position control (two axes). In addition, the disturbance due to the radial AMB can be further reduced by evenly distributing the magnetic flux from the radial AMB when it enters the axial AMB.

The proposed multi-hybrid AMB design is shown in Fig. 6. The magnetic loops generated by the PM and axial AMB are coupled in the silicon steel disc on the spindle. The magnetic loops generated by the axial and radial AMBs are coupled via the silicon steel layer on the spindle, as illustrated in Fig. 6(a). The radial AMB loop serves as the major loop so that part

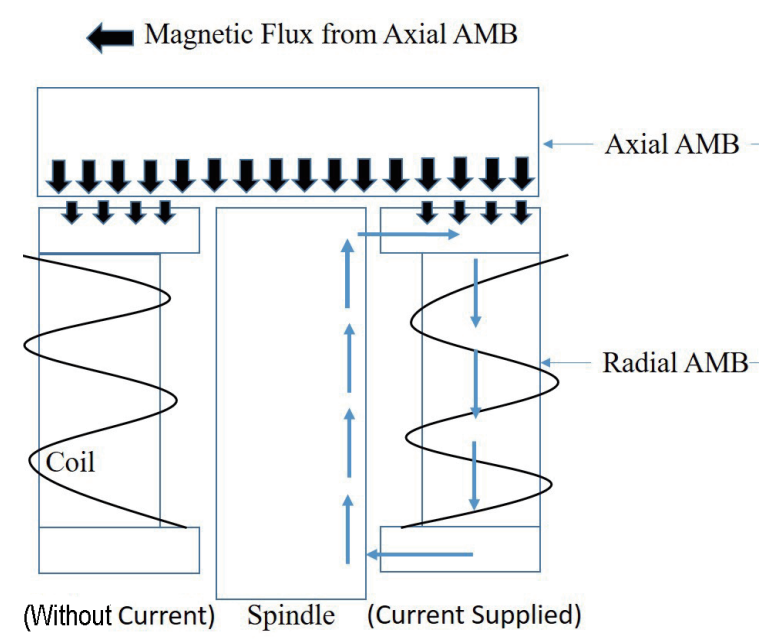

(a) $\longleftarrow$ Magnetic Flux from Radial AMB

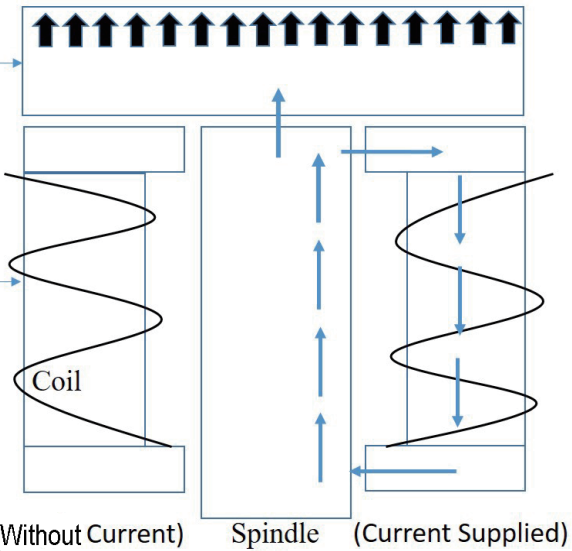

(b)

Fig. 5. (Color online) Coupled axial and radial magnetic loops: (a) major: axial AMB; minor: radial AMB; and (b) major: radial AMB; minor: axial AMB. 


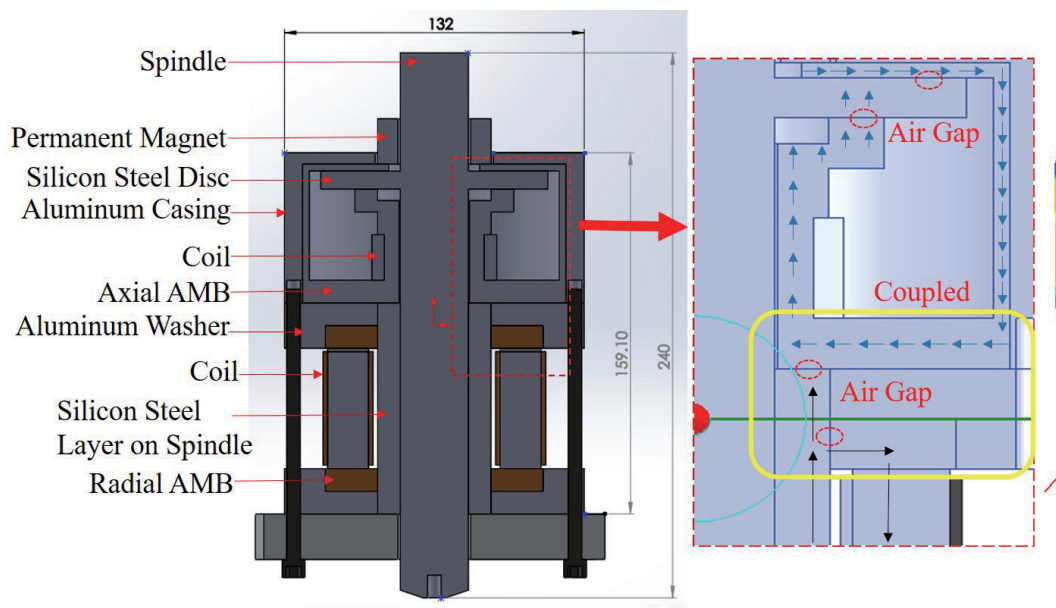

(a)

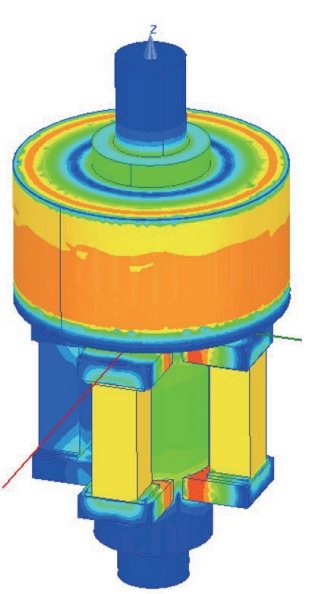

(b)

Fig. 6. (Color online) Proposed multi-hybrid AMB design: (a) cross-sectional view and (b) magnetic analysis result.

of the magnetic flux of the radial AMB will enter the axial AMB loop. On the other hand, the regulation force demand and the dimensions of the axial AMB could be downgraded owing to the assistance of the radial AMB. The magnetic analysis result in the case of two radial coils operating is shown in Fig. 6(b).

\section{Design and Manufacture of Multi-hybrid AMB}

The proposed multi-hybrid AMB structure is depicted in Fig. 7. The prototype and specifications of the AMB are shown in Fig. 8 and listed in Table 4, respectively.

\subsection{Performance verification of multi-hybrid AMB prototype}

The test rigs used to investigate the induced magnetic force and the rotor position regulation function of the proposed design are shown in Fig. 9. The PID control strategy was employed in these tests via the dSPACE DS1104 signal processing interface. The magnetic force check [Fig. 9(a)], was performed to inspect the force induced by the individual coil and for the coupled condition, such as the magnetic force differences between the four radial coils and the difference in force between the analysis results and practical conditions. The individual magnetic force induced by these five coils can easily exceed $100 \mathrm{~N}$ under 1 A current supply (measured using a JIHSENSE JS-300 load cell). The maximum error of the induced magnetic force among the four coils in the radial AMB is below 3\% and the maximum difference in magnetic force generated by the axial coil in the analysis results and practical tests is about $5 \%$. The test rig in Fig. 9(b) was used to verify the rotor position regulation performance. The rotor position deviation along different axes were measured using KEYENCE EX-305 gap sensors. The trajectory of the rotor position deviation without the motor equipped (static mode) is shown in Fig. 10. The 5-DOF rotor control was verified successfully. The test results at a rotor speed 


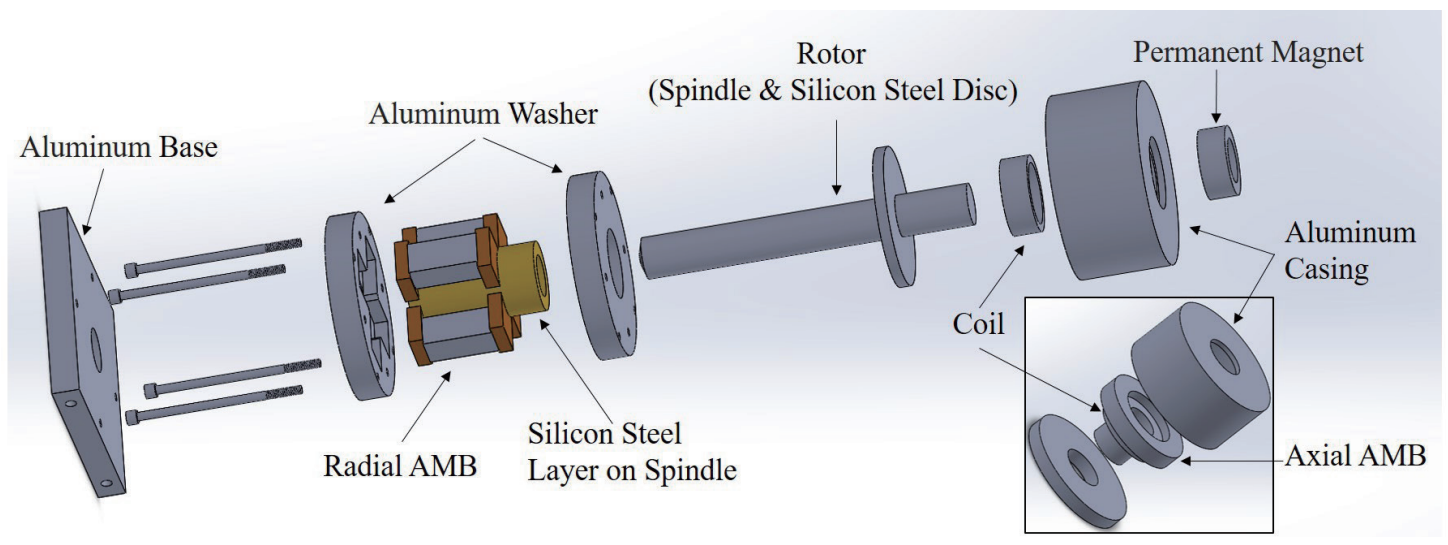

Fig. 7. (Color online) Multi-hybrid AMB structure proposed in this work.
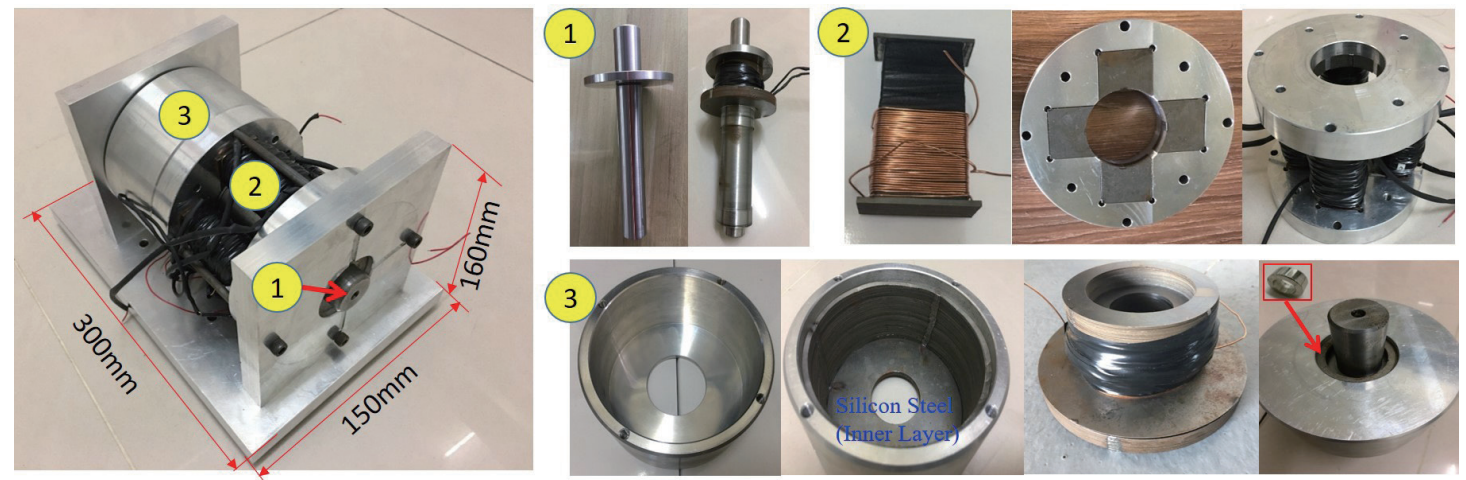

Fig. 8. (Color online) Prototype of multi-hybrid AMB.

Table 4

Specifications of multi-hybrid AMB prototype.

\begin{tabular}{|c|c|c|c|}
\hline Component or item & Material & Dimensions & Remark \\
\hline Axial AMB & - & $\varnothing 110 \times 58(\mathrm{~mm})$ & Outer size \\
\hline Radial AMB & - & $\varnothing 132 \times 97(\mathrm{~mm})$ & Outer size \\
\hline $\begin{array}{l}\text { Cross-sectional area } \\
\text { of I-shaped core }\end{array}$ & $\begin{array}{l}\text { Silicon steel } \\
35 \mathrm{H} 440\end{array}$ & $360 \mathrm{~mm}^{2}$ & Middle of I-shaped core \\
\hline $\begin{array}{l}\text { Cross-sectional area } \\
\text { of pole }\end{array}$ & $\begin{array}{l}\text { Silicon steel } \\
35 \mathrm{H} 440\end{array}$ & $794.59 \mathrm{~mm}^{2}$ & Radial AMB \\
\hline Spindle & Steel 1010 & $\varnothing 30 \times 240(\mathrm{~mm})$ & Main body of spindle \\
\hline Silicon steel layer & $\begin{array}{l}\text { Silicon steel } \\
35 \mathrm{H} 440\end{array}$ & $\varnothing 50 \times 93(\mathrm{~mm})$ & On spindle against radial AMB \\
\hline Rotor disc & $\begin{array}{l}\text { Silicon steel } \\
35 \mathrm{H} 440\end{array}$ & $\varnothing 100 \times 8(\mathrm{~mm})$ & On spindle against axial AMB \\
\hline $\mathrm{PM}$ & NdFe35 & $\varnothing 50 \times \varnothing 32 \times 10(\mathrm{~mm})$ & Ring type \\
\hline Washer and casing & Aluminum & - & - \\
\hline $\begin{array}{l}\begin{array}{l}\text { Coil } \\
\text { (single coil) }\end{array} \\
\end{array}$ & Copper & $\begin{array}{l}\text { Wire } \varnothing 0.7 \\
350 \text { turns }\end{array}$ & $\begin{array}{l}\text { One coil inside axial AMB and } \\
\text { four coils inside radial AMB }\end{array}$ \\
\hline Air gap & - & $\begin{array}{c}2 \mathrm{~mm} \text { between PM and spindle } \\
1 \mathrm{~mm} \text { between spindle and } \\
\text { radial AMB pole }\end{array}$ & Other air gaps are $0.15 \mathrm{~mm}$ \\
\hline
\end{tabular}




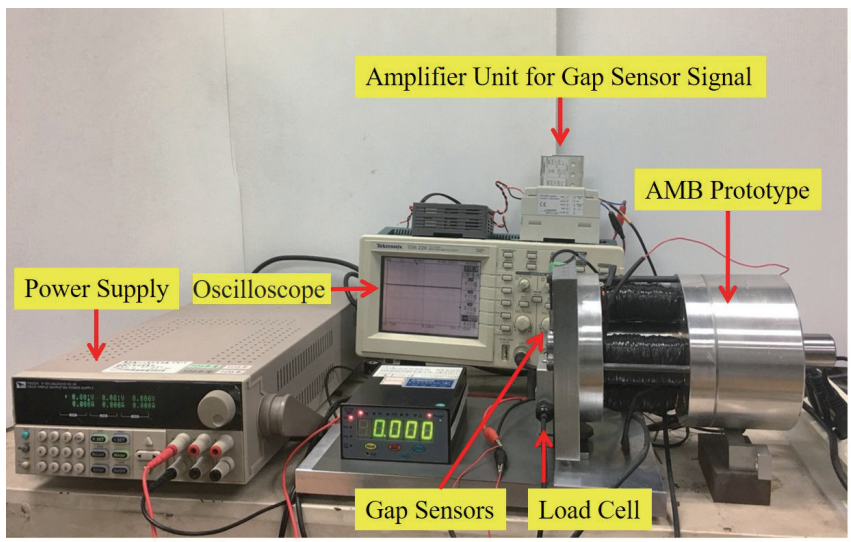

(a)

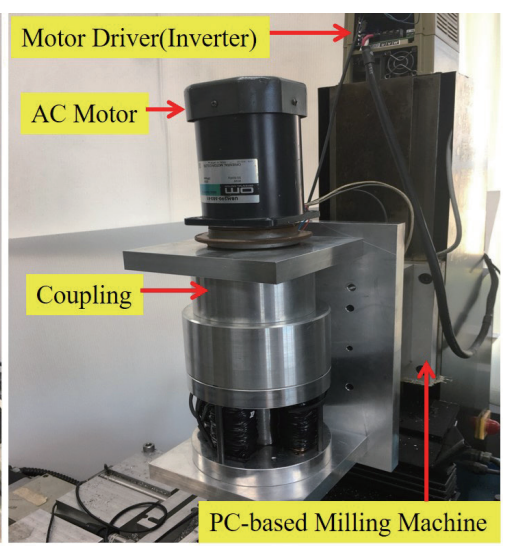

(b)

Fig. 9. (Color online) Test rigs for checking: (a) magnetic force and (b) rotor position regulation function.

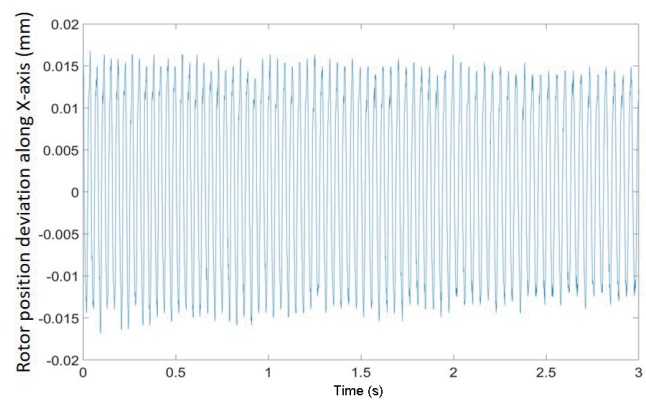

(a)

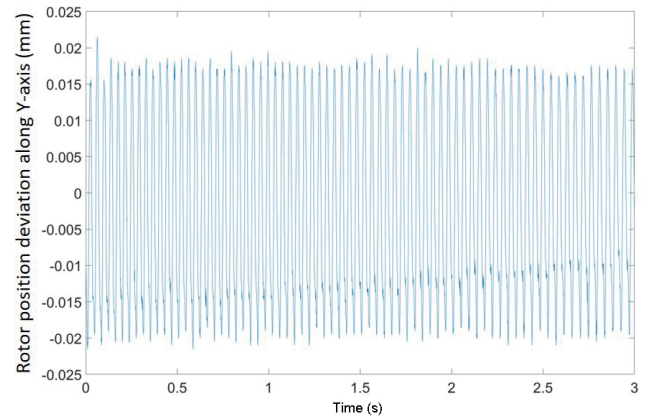

(c)

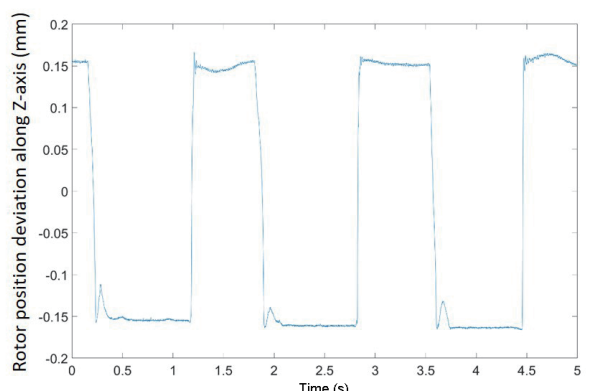

(b)

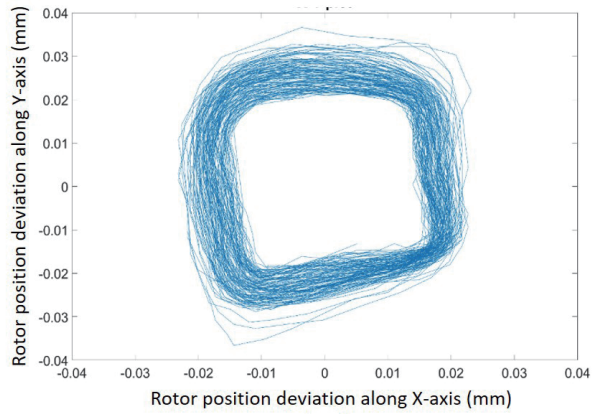

(d)

Fig. 10. (Color online) Static (without motor equipped) trajectory of rotor position deviation along (a) $X$-axis, (b) $Z$-axis, and (c) $Y$-axis, and in (d) $X-Y$ plane.

of $300 \mathrm{rpm}$ (dynamic mode) are shown in Fig. 11. Since the motor is equipped, the control of the axial coil is turned off (the axial rotor position deviation is no longer controllable). The large variation of the rotor trajectory at the beginning is due to the starting of the motor, and the rotor dynamics become stable within $1 \mathrm{~s}$. The maximum rotor position deviation under the dynamic mode is less than that under the static mode because of the different DOF of the rotor. 


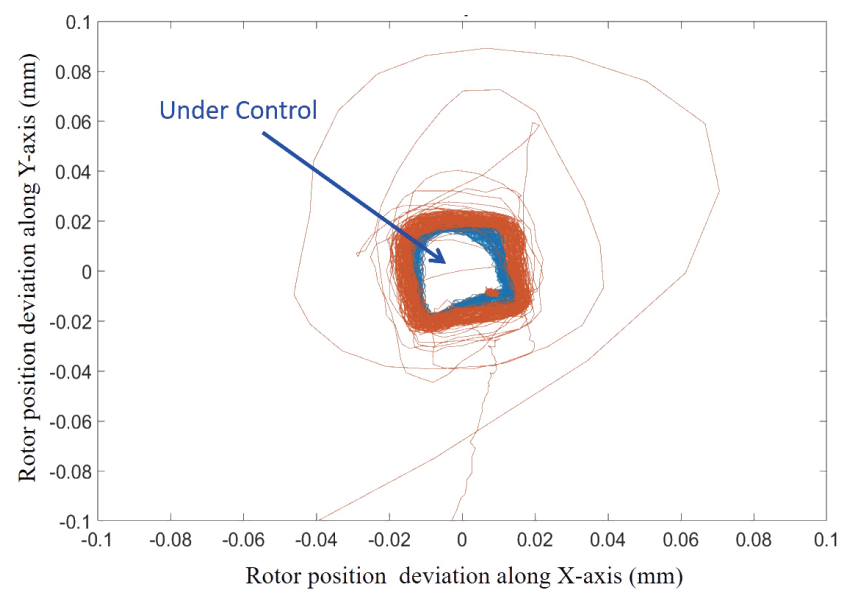

Fig. 11. (Color online) Trajectory of rotor position deviation at $300 \mathrm{rpm}$ in $X-Y$ plane.

\section{Conclusions}

A multi-hybrid AMB was designed, manufactured, and practically verified in this work. The term "multi-hybrid" means there are two AMB structures (axial and radial) and three magnetic loops (generated by two AMBs and one PM) involved and combined in this design. With the aid of specially designed magnetic channels, axial and radial rotor position regulation functions were synthesized and a compact prototype was accomplished. According to the experimental results under the static mode, the default function for the magnetic levitation of a 5-DOF rotor was successfully performed. The control precision of the 5-DOF rotor position deviation can be further improved by applying a high-performance control strategy. The proposed multi-hybrid AMB design can be applied to a 5-DOF milling spindle or robot arm joints. For the application of the 5-DOF milling spindle, concerns include the maximum magnetic regulation force, the speed of rotor position regulation, the maximum rotor position deviation, the combination of magnetic loops of the AMBs and the driving motor, and the function of active compensation against cutting vibrations. For robot arm joint applications, the size, weight, power consumption, maximum support force, regulation speed of the joint position, and maximum rotor position deviation will be the main performance indexes.

\section{Acknowledgments}

This research was supported by the Ministry of Science and Technology (Taiwan, under Grant MOST 108-2221-E-153-011) and National Pingtung University (under Grant NPTU-108003), to whom the authors would like to express their appreciation.

\section{References}

1 J.-S. Chen and K.-W. Chen: Int. J. Mach. Tools Manuf. 45 (2005) 1487.

2 Y. Kato, T. Yoshida, and K. Ohniwa: Electr. Eng. Jpn. 165 (2008) 69. 
3 M. Noh, W. Gruber, and D. L. Trumper: IEEE-ASME Trans. Mech. 22 (2017) 2308.

4 A. Smirnov, N. Uzhegov, T. Sillanpää, J. Pyrhönen, and O. Pyrhönen: IEEE Trans. Ind. Electron. 64 (2017) 9876.

5 H. S. Zad, T. I. Khan, and I. Lazoglu: IEEE Trans. Ind. Electron. 65 (2018) 2537.

6 Z. Shuyue, P. Wei, W. Caobing, and W. Jihao: Proc. Cryogenic Engineering Conf. Int. Cryogenic Materials Conf. (2017) 278.

7 H. S. Zad, T. I. Khan, and I. Lazoglu: IEEE Trans. Ind. Electron. 65 (2018) 4006.

8 L. Ai, G. Zhang, W. Li, G. Liu, N. Song, L. Xiao, and L. Lin: IEEE Trans. Appl. Supercond. 28 (2018) 1.

9 S. C. Mukhopadhyay, T. Ohjj, M. Iwahara, and S. Yamada: IEE Proc.: Electr. Power Appl. 146 (1999) 33.

10 H. Y. Kim and C. W. Lee: Mechatronics 16 (2006) 13.

11 M. H. Kimman, H. H. Langen, and R. H. M. Schmidt: Mechatronics 20 (2010) 224.

12 N.-C. Tsai and R.-M. Lee: Int. J. Adv. Manuf. Technol. 53 (2010) 93.

13 Z. Xie, K. Yu, L. Wen, X. Wang, and H. Zhou: Chin. J. Aeronaut. 27 (2014) 1619.

14 S. Xu and J. Fang: IEEE T. Magn. 50 (2014) 1.

15 A. Katyayn and P. K. Agarwal: Proc. Int. Conf. Advances in Mechanical, Industrial, Automation and Management Systems (2017) 1.

16 S. Xu, X. Liu, and Y. Le: IEEE Trans. Magn. 53 (2017) 1. 